\title{
Políticas educativas y desigualdades escolares: El caso del programa Plan de Mejora Institucional (PMI) en el conurbano bonaerense
}

Palabras-claves:

políticas educativas, desigualdades escolares, escuela secundaria.

Education policies and school inequalities:

The case of the Institutional Improvement Plan in Buenos Aires

Keywords

education policies, school inequalities,secondary school.

\section{Vanesa Romualdo}

La tesis de la maestría en Política y Gestión de la Educación de la Universidad Nacional de Luján defendida y aprobada en diciembre de 2017 que aquí se reseña se enmarcó en el proyecto "Derecho a la educación, inclusión y políticas sociales: los desafíos de la democratización escolar en la última década" radicado en la Universidad Nacional de General Sarmiento, financiado por el Programa Consenso del Sur 2014-2015 (Ministerio de Educación de la Nación) y dirigido por Nora Gluz (directora también de la tesis). El mismo se articuló con el proyecto UBACyT 2014-2017 “Políticas públicas y democratización de la educación. Sentidos, regulaciones y procesos en torno a la inclusión en la actual coyuntura latinoamericana" (en el cual la autora fue becaria UBACyT), con sede en el Instituto de Investigaciones en Ciencias de la Educación (IICE) de la Facultad de Filosofía y Letras de la Universidad de Buenos Aires y cuya directora fue Myriam Feldfeber.

La tesis estudia, recuperando los aportes de la sociología y la política educacional, los sentidos sobre las desigualdades escolares que se configuraron en las escuelas a partir de la implementación de las políticas educativas destinadas al nivel secundario en la Argentina contemporánea. Para ello analizamos qué perspectivas respecto de las desigualdades escolares subyacen al diseño de las políticas, las estrategias y recursos institucionales que se ponen en juego a la hora de intervenir sobre las mismas y las apropiaciones que de ellas hacen escuelas que atienden población en condición de vulnerabilidad.

Tomamos como caso un programa educativo diseñado en el marco de la extensión de la obligatoriedad a todo el nivel secundario a partir de la Ley de Educación
Nacional (LEN) 26206/2006: el Plan de Mejora Institucional (PMI), creado por Resolución CFE 88/o9. La particularidad del PMI radica en que a través de una estrategia común a todo el sistema se propone intervenir sobre las desigualdades escolares a partir del reconocimiento del modo como la organización escolar incide sobre ellas. Nuestra preocupación por lo institucional deriva de la centralidad que adquirió la discusión sobre la organización escolar en las denominadas "políticas de inclusión" en materia de educación durante el período 2006-2015 y de la relevancia que esta dimensión adquirió en los estudios académicos que analizan los procesos de producción y reproducción de desigualdades en el campo escolar. El PMI se constituyó desde el discurso oficial en una herramienta central para el cambio de la escuela secundaria y se configuró, a su vez, como programa de carácter universal, atributo que consideramos relevante por constituir un desplazamiento significativo respecto de los procesos de intervención estatal para garantizar la extensión de la obligatoriedad escolar instaurados con la reforma neoliberal de los años noventa que se fundaron en la focalización compensatoria.

La investigación se llevó adelante mediante una metodología de tipo cualitativo, siendo sus principales técnicas de construcción de datos el relevamiento documental, la entrevista semiestructurada y la observación, y el trabajo de campo se llevó a cabo durante 2014 y 2015 en cuatro escuelas secundarias comunes de dos distritos de elevada vulnerabilidad social del conurbano bonaerense (La Matanza y José C. Paz).

A nivel académico, nuestra intención fue aportar a los debates acerca de la capacidad de intervención de las políticas educativas sobre las desigualdades escolares desde una perspectiva sociológica que interpela a la escuela en el marco de la totalidad social, a las 
políticas educativas en términos de las relaciones de fuerza, los discursos, las reglas de juego y las institucionalidades que las materializan y a las prácticas escolares en el marco de las mediaciones institucionales que regulan las conductas.

Algunos de los supuestos teóricos de los que partimos para pensar el Estado y las políticas públicas sostienen la idea de que tanto la igualdad como la desigualdad social son un resultado institucional (Danani, 2008), es decir que las consecuencias sociales de las políticas públicas están mediadas por la configuración de la institucionalidad estatal y las acciones de los actores sociales. Concebimos a las políticas públicas (policies) como textos en la medida en que se tratan de representaciones codificadas y decodificadas de modo complejo según las disputas, experiencias y contextos que atraviesan las interpretaciones y reinterpretaciones de los sujetos y, al mismo tiempo, como discursos en el sentido foucaultiano del término, porque son prácticas que construyen objetos delimitando ciertas posibilidades para pensar y actuar (Ball, 1998). Creemos que no existe una relación lineal entre la formulación normativa de las políticas y programas educativos y su puesta en práctica. Por el contrario, consideramos que las políticas no se "adoptan" ni se "ejecutan" o "aplican", sino que se recontextualizan y se van transformando inevitablemente en su circulación por los distintos sectores y actores del sistema educativo (Ezpeleta, 2004). Destacamos, desde esta perspectiva, el carácter procesual y la insoslayable inscripción contextual de la implementación de las políticas públicas, lo que evidencia la naturaleza política de su construcción (Rockwell y Ezpeleta, 1987). Seguimos a Gluz y Rodríguez Moyano (2013) al considerar que, desde esta perspectiva, el análisis de la institución escolar como ámbito de recontextualización de la política es central para explicar los mecanismos de dominación y legitimación de las desigualdades sociales (Bourdieu, 2002) o su capacidad para transformarlas.

Entre los principales hallazgos de la tesis encontramos en primer lugar que, si bien a nivel normativo tanto nacional como jurisdiccional se reconoce la complejidad y multicausalidad de los factores que inciden en las desigualdades escolares, posición que diferencia las políticas del período analizado de las del período neoliberal de los años noventa, en los procesos de apropiación a nivel de las instituciones las transformaciones han sido más bien marginales. Respecto de los modos de apropiación del PMI por parte de las escuelas, si bien las estrategias desarrolladas reconocen los límites de la gramática de la escolaridad (Tyacky Cuban, 2001) para garantizar la escolarización de todos los jóvenes, solo despliegan mecanismos ad hoc que denuncian dichas limitaciones sin modificar sus causas.

Decidimos clasificar las prácticas llevadas a cabo en las escuelas seleccionadas para implementar el PMI en tres tipos a fin de facilitar su análisis: PMI como espacio ad hoc para la acreditación que fue la línea mayoritaria e incluyó aquellas actividades que se organizaron en torno a clases de apoyo para la aprobación de asignaturas evitando, la mayoría de las veces, instancias de evaluación como las mesas de exámenes; PMI como ámbito de expresión, participación y pertenencia institucional que fueron las actividades extracurriculares, en general de tipo lúdico-recreativo, focalizadas en propiciar el desarrollo de disposiciones a la participación y la mejora de las capacidades expresivas de los estudiantes; y PMI como recurso complementario, la línea en la que los recursos del programa se utilizaron para sostener o reforzar actividades que las escuelas necesitaban sin estar sus objetivos inmediatamente vinculados con los del PMI.

En la línea de PMI como espacio ad hoc para la acreditación, entre los sentidos sobre las desigualdades escolares que orientaron las prácticas de los docentes, encontramos, en primer lugar, una mirada economicista que valora positivamente la existencia de estas clases de apoyo por la posibilidad de acompañamiento gratuito que brindan a sus estudiantes $y$, en segundo lugar, encontramos perspectivas que consideran a la escuela como productora de desigualdades escolares debido a la forma que adopta la gramática de la escolaridad que provoca el fracaso escolar de muchos estudiantes y también a causa del ausentismo o la falta de compromiso individual de los mismos docentes. Las estrategias en esta línea en realidad apuntaron a responder a una de las expresiones que materializa las desigualdades escolares, como es el fracaso o la no presentación de los estudiantes a las mesas de exámenes de asignaturas previas, sin modificar sus posibles causas: el mecanismo de evaluación en sí, las prácticas pedagógicas docentes o su ausentismo, los procesos de enseñanza-aprendizaje.

En lo que respecta a las concepciones sobre las desigualdades escolares que subyacen a las estrategias de PMI como ámbito de expresión, participación y pertenencia institucional, consideramos que, si bien en todas ellas hay un registro del origen social de las desigualdades escolares, buscan trabajar sobre los sujetos educandos y no sobre el trabajo escolar ni la organización escolar. Incluso en algunos casos encontramos una preocupación tan enfocada al impacto en lo individual que obtura la posibilidad de ver lo que aporta la escuela como institución y el contexto 
social a la producción de las desigualdades escolares produciéndose una psicologización de las relaciones sociales que singulariza a los estudiantes y convierte mecanismos como la escucha de los docentes en un método de control o pacificación social.

Por último, las estrategias que agrupamos bajo el nombre de PMI como recurso complementario no se preocupan particularmente por las desigualdades escolares entre los estudiantes, sino que funcionan más como una solución temporal a determinadas necesidades de las escuelas que no implica ningún tipo de modificación en la organización escolar ni en el puesto de trabajo docente o el trabajo escolar. Sin embargo, el uso que se les da a los recursos en las estrategias enmarcadas en esta línea da cuenta de la fragmentación educativa como expresión de las desigualdades escolares.

Encontramos, además, algunos elementos y situaciones condicionantes de la implementación del programa PMI en las cuatro escuelas analizadas. En primer lugar, la ubicación de las escuelas en barrios marginales y los problemas materiales y de infraestructura de algunas de ellas que conllevan limitaciones prácticas concretas. En lo que concierne a los modos de organización del trabajo de los docentes en el programa, hallamos una monopolización de las tomas de decisiones referidas al PMI por parte de los directivos, quienes incluso seleccionan a los docentes participantes del mismo, combinado con dificultades para trabajar colectivamente tanto entre los docentes que son parte del programa como con los que no, lo cual es una fuente de conflictos entre colegas. Muchos docentes que no participaron del PMI manifestaron su desacuerdo con que se evadieran las mesas de exámenes o que a sus alumnos "los evaluaran otros" o que incluso los aprobaran cuando ellos consideraban que no estaban en condiciones. A todo esto, se sumaron las condiciones salariales precarias que ofrece el programa a los docentes y la falta de acompañamiento y capacitación por parte del Estado que dejó librados a la voluntad y posibilidad de los actores los cursos de acción, lo cual se contradice con el reconocimiento de las desigualdades escolares existentes y la fragmentación educativa del marco normativo. Por otra parte, en cuanto a las representaciones de los docentes acerca del PMI, nos encontramos que la mayoría ve al programa como un "parche", una solución temporal a ciertas preocupaciones, y coincide en que este no interviene sobre las verdaderas causas de los problemas educativos como las prácticas docentes y la organización escolar, aspectos que el programa no modificó estructuralmente.

Finalmente, en lo que respecta a las dinámicas que se configuraron con la implementación del PMI, encontramos tensiones entre una tendencia hacia una mayor atención a las trayectorias escolares diversas y el acotamiento de los tiempos que implicó la corta duración de las actividades del programa, lo que además conllevó una reducción de los contenidos curriculares a enseñar. Las oportunidades de enseñanza individualizada abiertas por lo que denominamos pedagogía de la proximidad y por la mayor permanencia de los estudiantes en las escuelas entran en contradicción con la enseñanza de menos contenidos, su simplificación o la prevalencia de la recompensa de actitudes en lugar de progresos académicos, lo que podría estar abonando a la reproducción de desigualdades escolares más que a su superación. Estos nudos críticos que aparecen como problemáticas en las cuatro escuelas analizadas, si bien no tienen pretensión de generalización alguna, evidencian algunas de las consecuencias sociales de las políticas educativas recientes y es en este sentido que la tesis deja abiertos distintos interrogantes para seguir investigando. 


\section{Referencias bibliográficas}

»Ball, S. (1998). Big Policies/Small World: An introduction to international perspectives in education policy. En Comparative Education, vol. 34, núm. 2, pp. 119-130.

"Congreso Nacional de Argentina. (14 de diciembre de 2006). Ley de Educación Nacional. [Ley 26206 de 2006].

»CFE, Resolución 88/o9.

" Bourdieu, P. (2002). La distinción. Criterios y bases sociales del gusto. Madrid, Taurus.

»Danani, C. (2008). América Latina luego del mito del progreso neoliberal: las políticas sociales y el problema de la desigualdad. En Ciências Sociais Unisinos, janeiro/abril.

»Ezpeleta Moyano, J. (2004). Innovaciones educativas. Reflexiones sobre los contextos en su implementación. Revista Mexicana de Investigación Educativa, 9, 403-424.

" Gluz, N. y Rodríguez Moyano, I. (2013). Asignación Universal por Hijo, condiciones de vida y educación. Las políticas sociales y la inclusión escolar en la provincia de Buenos Aires. Archivos Analíticos de Políticas Educativas, 21 (21).

» Rockwell, E. y Ezpeleta, J. (1987). La escuela: relato de un proceso de construcción inconcluso. En $F$. Madeira y G. Namo de Mello (Coords.), Educação na América Latina, Os modelos teóricos e realidade social, Sao Paulo: Cortez Editora-Editora Autores Asociados.

» Tyack, D. y Cuban, L. (2001). En busca de la utopía. Un siglo de reformas de las escuelas públicas. [Traducción al español de Mónica Utrilla]. México, FCE.

\section{Breve currículum de la autora}

\section{Vanesa Romualdo}

Doctoranda en Ciencias Sociales por la Universidad de Buenos Aires (UBA). Magíster en Política y Gestión de la Educación por la Universidad de Luján (UNLu). Licenciada y profesora en Sociología (UBA). Actualmente becaria de investigación UBACyT en el Instituto de Investigaciones en Ciencias de la Educación (IICE-UBA). Docente de la cátedra de Sociología de la Educación en la Facultad de Ciencias Sociales, UBA, Argentina. Correo electrónico: vanesa.romualdo@gmail.com 